\title{
Benefício da inteligência artificial para o diagnóstico precoce da cárie dentária:
}

\section{Revisão integrativa}

\author{
Effectiveness of artificial intelligence in the treatment of dental caries: An integrative review
}

\section{Efectividad de la inteligencia artificial en el tratamiento del diagnóstico de caries: Una revisión} integradora

\section{Resumo}

A inteligência artificial (doravante IA ou em inglês AI - artificial intelligence) é um ramo da ciência da computação que utiliza algoritmos semelhantes a um especialista no ato de tomar decisões e resolver problemas complexos. Na saúde, os benefícios vêm identificando melhores tratamentos e ajudando na prevenção e detecção de doenças em estágio inicial. O objetivo deste trabalho foi realizar uma revisão integrativa da literatura atual sobre IA para o diagnóstico precoce em lesões de cárie, considerando os benefícios, limitações e impacto para a sociedade e para a saúde bucal. Uma revisão integrativa da literatura foi realizada por meio de uma busca de 6 artigos científicos em inglês nas bases de dados eletrônicas: PubMed, Cochrane, Scopus, e Web of Science, utilizando os descritores: cáries dentais, inteligência artificial e odontologia. Além disso, 6 outros artigos foram encontrados por meio de busca manual, indexados no período de 2016 a 2020, que tratavam estudos de relato de caso, estudo in vitro e in vivo. Após os critérios de elegibilidade, foram analisados 12 artigos integralmente publicados em língua inglesa e língua portuguesa. Os estudos mostraram que os vários métodos atuais da inteligência artificial oferecem mais precisão, facilidade no diagnóstico e eficácia no tratamento. Contudo, essa técnica está passando por uma fase experimental, necessitando de aprimoramento para diminuir a probabilidade de erros e equívocos causados por esse sistema.

Palavras-chave: Cárie dentária; Inteligência artificial; Odontologia.

\section{Abstract}

The artificial intelligence (AI) is a branch of computing science which uses similar algorithms as a specialist before making any decisions and solving complex issues. In health, the benefits occur from data generation to improve 
management processes, identifying better treatments and helping in prevention and disclosure early-stage diseases. The purpose of this survey was to perform an integrative review on modern literature about AI to early diagnosis caries injuries, considering the benefits, limitations and impact in society and oral health. An integrative review had been done by searching 6 English scientific articles using on-line databases: PubMed, Cochrane, Scopus and Web of Science, using keywords such as: dental caries, artificial intelligence and dentistry. Moreover, other 6 articles were found by manual research indexed from 2016 to 2020, which worked with case report titled in vitro and in vivo. After eligibility criteria, 12 articles fully published in English and Portuguese were analyzed. The studies have shown that several modern methods of artificial intelligence offered exactness, ease of diagnosis and treatment effectiveness. Nevertheless, this technique has been through an experimental phase, it is required enhancement to reduce mistakes and misconception caused by this system.

Keywords: Dental caries; Artificial intelligence; Dentistry.

\section{Resumen}

La Inteligencia artificial denominada IA o en inglés AI (artificial intelligence) es un ramo de la ciencia de la computación que utiliza los ritmos semejantes a un especialista en el acto de tomar decisiones y resolver problemas complejos. En la salud los benefícios vienen por la generación de datos para la optimización de procesos en la gestión, identificando mejores tratamientos y ayudando en la prevención y detección de enfermedades en su etapa inicial. El objetivo de este trabajo fue realizar una revisión integral de la literatura actual sobre la IA para el diagnóstico precoz en lesiones de caries, considerando los beneficios, las limitaciones y el impacto para la sociedad y para la salud bucal. Una revisión integral de la literatura fue realizada por medio de una búsqueda de 6 artículos científicos en inglés en bases de datos electrónicas: PubMed, Cochrane, Scopus, y Web of Science, utilizando los descriptores: caries dental, Inteligencia artificial y odontologia. Además de esto, otros 6 artículos fueron encontrados a través de búsqueda manual indexados en el período de 2016 a 2020 que trataban de estudios de informes de caso, estudio in vitro e in vivo. Posteriormente a los criterios de elegibilidad, fueron analizados 12 artículos íntegramente publicados en lengua inglesa y lengua portuguesa. Los estudios mostraron que los varios métodos actuales de Inteligencia artificial ofrecen más precisión, facilidad en el diagnóstico y eficacia en el tratamiento. Sin embargo, esta técnica está pasando por una fase experimental, necesitando optimización para diminuir la probabilidad de errores y equívocos causados por este sistema.

Palabras clave: Caries dental; Inteligencia artificial; Odontología.

\section{Introdução}

A cárie dentária é uma das condições clínicas predominantes no mundo, afetando principalmente pessoas de 6 a 19 anos. Produzem ácidos que danificam o esmalte dental e ocorre quando as bactérias orais metabolizam açúcares e amidos encontrados nos alimentos. Para localizar e estabelecer a profundidade da cárie, cirurgiões dentistas utilizam radiografias, simultaneamente com o exame visual de luz branca. As radiografias tradicionais têm baixa sensibilidade para lesões e os detalhes mais finos muitas vezes não aparecem. (Angelino et al. 2017). Cruz et al. (2020) considera que é necessário realizar estudos que busquem tecnologias mais aprimoradas na realização dos métodos convencionais ou métodos novos, que sejam capazes de avaliar de forma mais precisa as lesões de cárie.

Sendo assim, uma das técnicas discutidas pelos autores é o desenvolvimento do Near - Infrared, (NIR), um sistema de imagem LED NIR utilizado para obter imagens dos dentes que apresentam esmalte dentário sadio ou não, com objetivo de identificar a presença de lesão cariosa de maneira eficaz. Essa técnica melhorou em alguns aspectos quando relacionadas com as radiografias bidimensionais, já que não possui radiação ionizante e contém imagens detalhadas, concedendo maior poder de diagnóstico. Com as imagens capturadas pelo sistema NIR, é possível detectar condições clínicas de forma mais fácil, introduzilo de maneira mais rápida e com um menor custo. (Angelino et al. 2017). Segundo Araújo et al. (2020), para que seja um sistema ideal, é necessário obter um diagnóstico precoce e em estágio inicial na detecção de cárie, fazendo com que o cirurgião dentista faça um plano de tratamento adequado e melhore a tomada de decisão.

Além do NIR, outras metodologias que podem gerar benefícios para a saúde bucal vêm sendo desenvolvidas, as redes neurais convolucionais (CNNs). (Angelino et al. 2017). Essas redes, além de auxiliar os dentistas na avaliação de imagens de maneira rápida, também ajudam na detecção de estruturas e patologias (Endres et al. 2020). As imagens que são capturadas pelo sistema de imagem LED NIR passam por um processo de treinamento através das CNNs, emitindo um diagnóstico preciso e 
efetivo. (Angelino et al. 2017). Entretanto, ainda há limitações, já que essas técnicas estão em fase experimental, necessitando de aprimoramento para diminuir a probabilidade de erros e equívocos causados pelo sistema.

Desta forma, este estudo visa realizar uma revisão integrativa da literatura para investigar a eficácia da inteligência artificial no tratamento do diagnóstico da cárie, destacando benefícios, limitações e impacto para sociedade e para saúde bucal.

\section{Metodologia}

\subsection{Estratégia de Busca}

O presente estudo trata-se de uma revisão integrativa da literatura, com qualidade metodológica de uma revisão sistemática, é um estudo secundário, baseado em estudos primários, para resumir os resultados de estudos publicados sobre o benefício da inteligência artificial e a sua eficácia no diagnóstico da cárie. O trabalho foi realizado por meio de uma busca de artigos científicos na base de dados eletrônicos PubMed, Scopus, Cochrane e Web of Science, utilizando os descritores "cárie dentaria", "terapia a laser", "radioterapia" combinados com as associações e desfechos de interesse foram elaborados entre os descritores descritos abaixo nas Figuras 1, Figura 2, Figura 3 e Figura 4. Além das buscas nas bases de dados, também houve uma busca manual. A metodologia é do tipo qualitativa, utilizando o método de coleta por amostragens, segundo referenciado por Pereira et al. (2018).

Figura 1. Fluxograma de estratégia de busca nas bases de dados PubMed, Scopus, Cochrane e Web of Science.

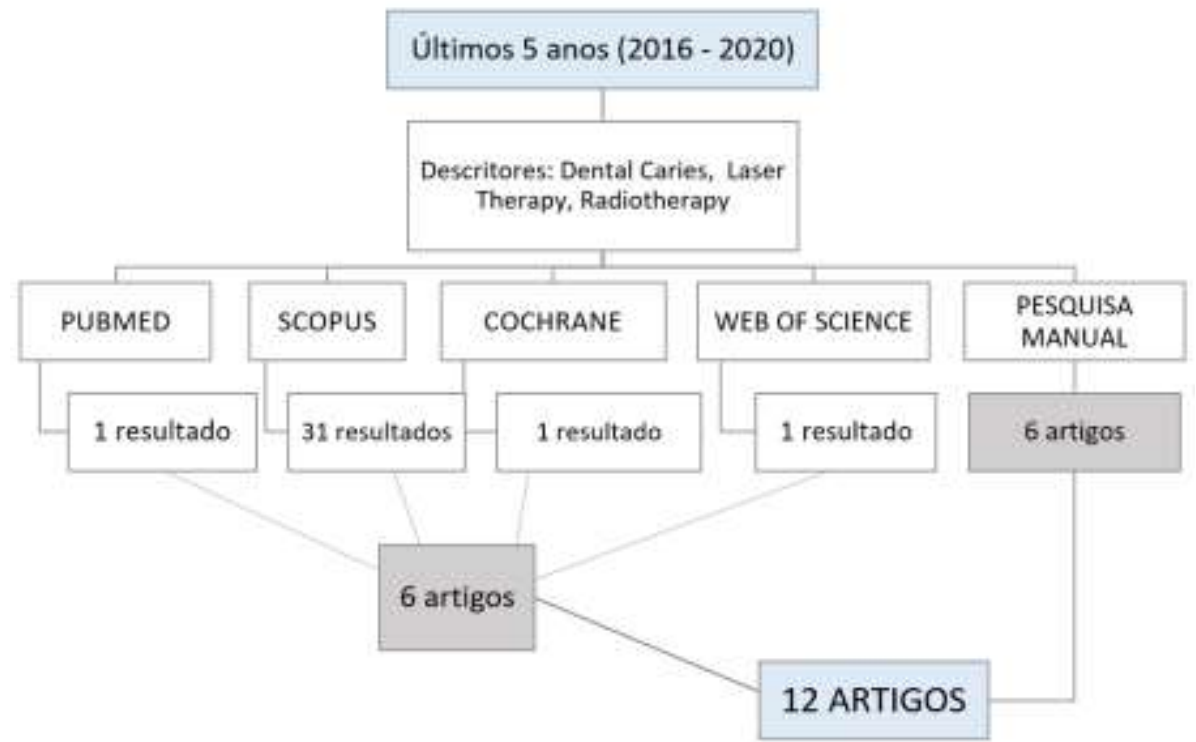

Fonte: Autores (2020).

A partir da busca de artigos científicos nas plataformas acima mencionadas, no período de 2016 a outubro de 2020, utilizando o descritor "dental caries" obtivemos um total de 9.927 artigos no PubMed; 1.240 no Scopus; 52 no Cochrane e 3.802 no Web of Science. Após a junção desse descritor com "artificial intelligence", foram filtrados 131 artigos no PubMed; 152 no Scopus; 7 no Cochrane e 16 no Web of Science, resultados que já apareciam na pesquisa com apenas o primeiro descritor. Após a junção dos descritores anteriores com "dentistry” obtivemos uma amostra de artigos 1 no PubMed; 31 no Scopus; 1 Cochrane e 1 no Web of Science.

Como foi mencionado acima, houve também uma busca manual e foram encontrados 6 artigos. Essa busca foi feita através de uma investigação nas referências dos artigos que foram obtidos nas plataformas oficiais citadas acima. 


\subsection{Seleção das publicações e extração dos dados}

O processo de análise para avaliação e seleção dos artigos foi realizado por dois pesquisadores, de forma independente, com posterior confronto dos resultados para obtenção dos textos selecionados por consenso. Os artigos indexados repetidamente nos quatro bancos de dados foram considerados apenas uma vez. Então, a seleção das publicações foi conduzida em duas fases:

(1) leitura dos resumos e (2) análise qualitativa dos textos na íntegra.

\subsection{Critérios de elegibilidade dos artigos}

Para seleção das fontes, foram consideradas como critérios de inclusão dos artigos originais disponibilizados na íntegra, publicados em língua inglesa, indexados no período de 2016 a outubro de 2020 que tratavam de estudos in vitro, estudos in vivo, revisão narrativa, revisão sistemática, revisão de escopo e revisão crítica associadas ao diagnóstico da cárie e inteligência artificial. Não foram incluídos artigos de revisão de literatura, relato de caso de diagnóstico de cárie guiada para outras terapias, publicadas em outras línguas ou em um período diferente do delimitado. Segue a Figura 2 que ilustra a estratégia de busca de acordo com os critérios citados.

Figura 2. Fluxograma de estratégia de busca e seleção dos artigos.

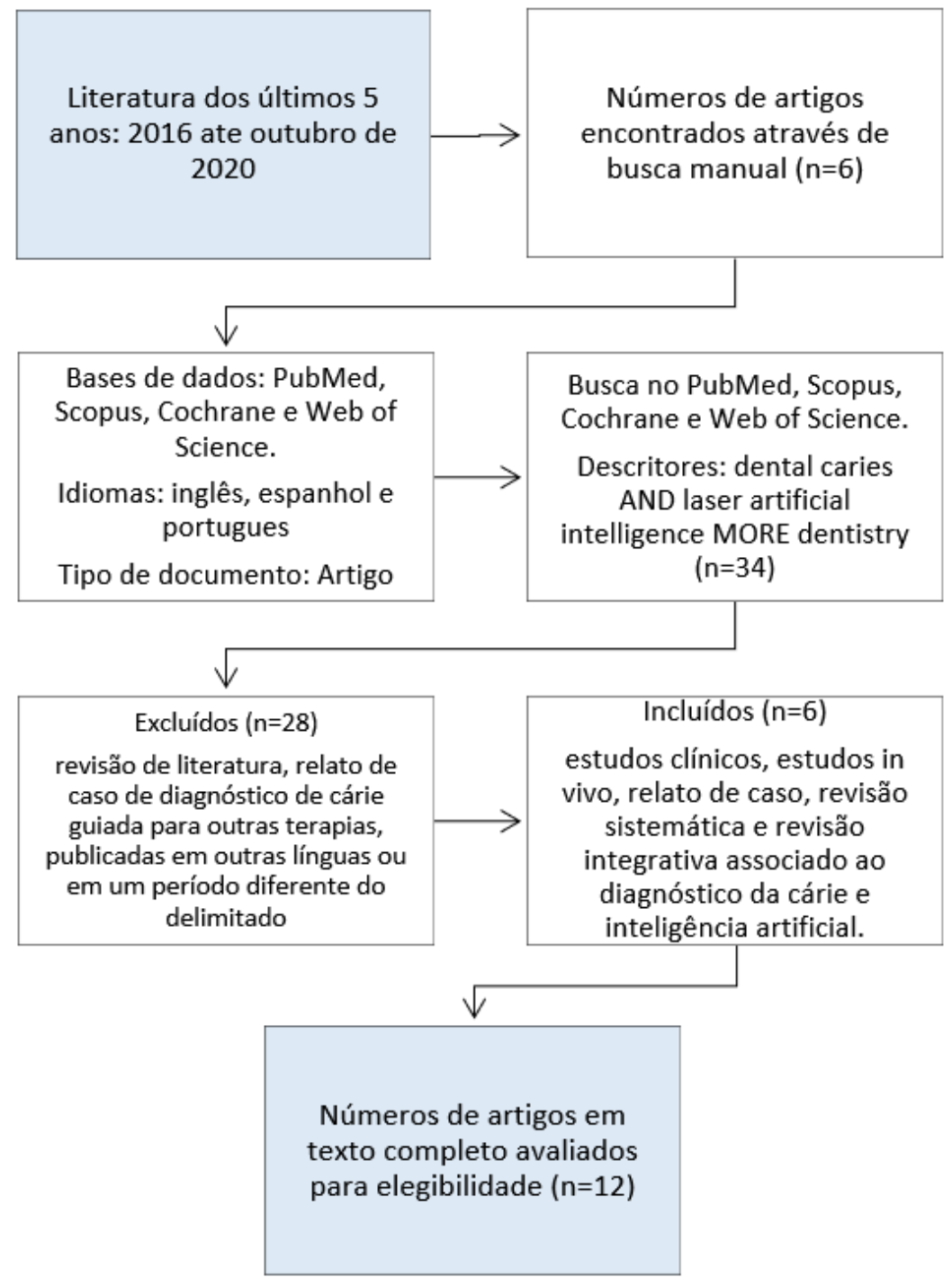


Essa metodologia seguiu as recomendações PRISMA (principais itens para relatar revisões sistemáticas e metanálise), como mostra na Figura 2. Os artigos que não se encaixavam nos critérios de inclusão, ou repetidos, foram excluídos. Posteriormente, dos artigos restantes, foi realizada a leitura completa, chegando a uma amostra final de 12 artigos incluídos nesta revisão.

Não foram utilizadas teses, dissertações e monografias, visto que a realização de uma busca desses estudos é inviável logisticamente e a base de dados utilizada não tem esse tipo de publicação.

\section{Resultados e Discussões}

Este estudo visou analisar a literatura a respeito dos benefícios, limitações, impacto e relevância do uso da inteligência artificial para diagnóstico precoce das lesões cariosas. A presente revisão integrativa foi capaz de encontrar estudos recentes que trouxeram avanços tecnológicos para a saúde bucal, mostrando metodologias que são capazes de predizer o surgimento de lesões cariosas e de identificar dados de forma precisa, rápida e eficaz, como mostra no Quadro 1.

Quadro 1. Seleção dos artigos de acordo com o autor, tipo de estudo, objetivos, metodologia, resultados e conclusão.

\begin{tabular}{|c|c|c|c|c|}
\hline Author & Objective & Metodology & Results & Conclusion \\
\hline $\begin{array}{l}\text { ANGELINO, K. } \\
\text { et al. } \\
(2017) \\
\text { In vivo }\end{array}$ & $\begin{array}{l}\text { We report the construction } \\
\text { of an NIR }(850 \mathrm{~nm}) \text { LED } \\
\text { imaging system, comprised } \\
\text { of an NIR source and an } \\
\text { intraoral camera for rapid } \\
\text { dental evaluations. }\end{array}$ & $\begin{array}{l}\text { The NIR system was composed } \\
\text { primarily of two intraoral } \\
\text { wands. An } 850 \text { nm LED was } \\
\text { embedded in to be used as the } \\
\text { source illumination. During } \\
\text { operation, the NIR system was } \\
\text { connected to a computer and a } \\
\text { generic webcam application } \\
\text { launched; live video feed was } \\
\text { displayed onscreen. }\end{array}$ & $\begin{array}{l}\text { The images captured by the } \\
\text { NIR system revealed } \\
\text { clinical conditions, } \\
\text { including caries, } \\
\text { demineralization, fractures, } \\
\text { cracks, and craze lines. The } \\
\text { detail afforded by NIR } \\
\text { images offers the possibility } \\
\text { of preemptive. }\end{array}$ & $\begin{array}{l}\text { In a study of } 10 \text { subjects } \\
\text { with varying dental } \\
\text { conditions, we test a } \\
\text { custom NIR system } \\
\text { capable of illuminating } \\
\text { at } 850 \mathrm{~nm} \text { and imaging } \\
\text { with a standard CMOS } \\
\text { USB camera. }\end{array}$ \\
\hline $\begin{array}{l}\text { LEÃO FILHO, } \\
\text { J.C.B. et al. } \\
\text { (2017) } \\
\text { In vitro }\end{array}$ & $\begin{array}{l}\text { Facilitar o diagnóstico de } \\
\text { lesões incipientes de cárie } \\
\text { com o desenvolvimento de } \\
\text { métodos atualizados, } \\
\text { suprindo a deficiência dos } \\
\text { métodos convencionais. }\end{array}$ & $\begin{array}{c}\text { Esses novos métodos são } \\
\text { baseados na mensuração da } \\
\text { diferença de condutividade } \\
\text { elétrica entre tecido sadio e } \\
\text { tecido lesado, em imagens } \\
\text { obtidas através de radiação X e } \\
\text { na dispersão da luz visível nos } \\
\text { tecidos dentais. }\end{array}$ & $\begin{array}{l}\text { O desenvolvimento de } \\
\text { métodos para a detecção de } \\
\text { lesões incipientes de cárie, } \\
\text { para ajudar no diagnóstico e } \\
\text { acompanhamento da } \\
\text { progressão dessas lesões, é } \\
\text { crítico para suprir as } \\
\text { necessidades clínicas, } \\
\text { devido ao surgimento de um } \\
\text { novo perfil de cárie. }\end{array}$ & $\begin{array}{c}\text { Com compreensão } \\
\text { crescente do processo } \\
\text { da cárie dental, a criação } \\
\text { de sistemas diagnósticos } \\
\text { sensíveis e específicos é } \\
\text { necessária para permitir } \\
\text { a detecção precoce de } \\
\text { desmineralização. }\end{array}$ \\
\hline $\begin{array}{l}\text { LEE, J.H. et al. } \\
\text { (2018) } \\
\text { In vitro }\end{array}$ & $\begin{array}{l}\text { The aim of the current study } \\
\text { was to evaluate the efficacy } \\
\text { of deep CNN algorithms for } \\
\text { detection and diagnosis of } \\
\text { dental caries on periapical } \\
\text { radiographs. }\end{array}$ & $\begin{array}{l}\text { A total of } 3000 \text { periapical } \\
\text { radiographic images were } \\
\text { divided into a training and } \\
\text { validation dataset }(n=2400 \\
[80 \%]) \text { and a test dataset } \\
(n=600[20 \%]) \text {. A pre-trained } \\
\text { GoogLeNet Inception v3 CNN } \\
\text { network was used for } \\
\text { preprocessing and transfer } \\
\text { learning. }\end{array}$ & $\begin{array}{l}\text { The premolar model } \\
\text { provided the best AUC, } \\
\text { which was significantly } \\
\text { greater than those for other } \\
\text { models }(P<0.001) \text {. }\end{array}$ & $\begin{array}{l}\text { This study highlighted } \\
\text { the potential utility of } \\
\text { deep CNN architecture } \\
\text { for the detection and } \\
\text { diagnosis of dental } \\
\text { caries. }\end{array}$ \\
\hline $\begin{array}{l}\text { HUNG, M. et } \\
\text { al. (2019) } \\
\text { In vivo }\end{array}$ & $\begin{array}{l}\text { This study sought to utilise } \\
\text { machine learning methods } \\
\text { in artificial intel- ligence to } \\
\text { select the most relevant } \\
\text { variables in classifying the } \\
\text { presence and absence of } \\
\text { root caries. }\end{array}$ & $\begin{array}{l}\text { Several supervised machine } \\
\text { learning methods were applied } \\
\text { to construct a tool that was } \\
\text { capable of classifying variables } \\
\text { into the presence and absence of } \\
\text { root caries. }\end{array}$ & $\begin{array}{l}\text { Of the machine learning } \\
\text { algorithms developed, } \\
\text { support vector machine } \\
\text { dem- onstrated the best } \\
\text { performance with an } \\
\text { accuracy of } 97.1 \% \text {, } \\
\text { precision of } 95.1 \% \text {, sen- } \\
\text { sitivity of } 99.6 \% \text { and } \\
\text { specificity of } 94.3 \% \text { for } \\
\text { identifying root caries. }\end{array}$ & $\begin{array}{l}\text { The machine learning } \\
\text { algorithms developed in } \\
\text { this study perform well } \\
\text { and allow for clinical } \\
\text { implementation and } \\
\text { utilisation by dental and } \\
\text { nondental professionals. }\end{array}$ \\
\hline
\end{tabular}




\begin{tabular}{|c|c|c|c|c|}
\hline $\begin{array}{l}\text { PINGALI, L. } \\
\text { (2019) } \\
\text { In vitro }\end{array}$ & $\begin{array}{l}\text { to create a cost-effective, } \\
\text { multimodal, personal oral } \\
\text { sensing device that } \\
\text { automatically classifies } \\
\text { sensed data and provides } \\
\text { interactive advice about oral } \\
\text { health. }\end{array}$ & $\begin{array}{l}\text { This project selected a camera } \\
\text { that has a convenient size for } \\
\text { easy placement in the mouth, } \\
\text { has a focal length of } 3 \mathrm{~cm} \text { or } \\
\text { less, sufficient magnification, } \\
\text { adjustable LED lighting, } \\
\text { connects to both Smartphones } \\
\text { and PCs, has a periscope mirror } \\
\text { to enable pointing at the right } \\
\text { angle, and is waterproof. }\end{array}$ & $\begin{array}{l}\text { It was noted that POHA } \\
\text { consistently correctly } \\
\text { classified the teeth of the } \\
\text { family members as not } \\
\text { having cavities or gingivitis } \\
\text { in } 200 \text { trials and also } \\
\text { correctly detected the cavity } \\
\text { that one family member had } \\
\text { in } 100 \text { trials. }\end{array}$ & $\begin{array}{l}\text { The results were } \\
\text { promising and showed } \\
\text { that it is indeed possible } \\
\text { to build a Personal Oral } \\
\text { Health Advisor using } \\
\text { multimodal sensors that } \\
\text { can be used by people at } \\
\text { homes. }\end{array}$ \\
\hline $\begin{array}{l}\text { SCHWENDIC } \\
\text { KE, F. et al. } \\
\quad(2019) \\
\text { Scope review }\end{array}$ & $\begin{array}{c}\text { A scoping review was } \\
\text { carried out, exploring use } \\
\text { cases, methodologies and } \\
\text { findings of studies applying } \\
\text { CNN on dental image } \\
\text { material. }\end{array}$ & $\begin{array}{l}\text { The most commonly used } \\
\text { image type were panoramic } \\
\text { radiographs }(\mathrm{n}=11), \\
\text { followed by periapical } \\
\text { radiographs }(\mathrm{n}=8) \text {, Cone- } \\
\text { Beam CT or conventional CT (n } \\
=6 \text { ). }\end{array}$ & $\begin{array}{l}\text { A comparison of the CNN } \\
\text { performance against an } \\
\text { independent test group of } \\
\text { dentists was provided by } \\
\text { seven } \\
\text { studies; most studies found } \\
\text { the CNN to perform similar } \\
\text { to dentists. }\end{array}$ & $\begin{array}{l}\text { CNNs are increasingly } \\
\text { employed for dental } \\
\text { image diagnostics in } \\
\text { research settings. }\end{array}$ \\
\hline $\begin{array}{l}\text { ARAÚJO, A.A. } \\
\text { et al. (2020) } \\
\text { Revisão } \\
\text { narrativa }\end{array}$ & $\begin{array}{l}\text { Revisar, na literatura, quais } \\
\text { são as ferramentas } \\
\text { disponíveis para o } \\
\text { diagnóstico e detecção da } \\
\text { cárie e suas lesões. }\end{array}$ & $\begin{array}{c}\text { Há diversos métodos de } \\
\text { detecção, como: ICDAS } \\
\text { (International Caries Detection } \\
\text { and Assessment System), } \\
\text { Caries Assessment Spectrum } \\
\text { and Treatment - CAST), } \\
\text { DIAGNOdent@ - LF; } \\
\text { DIAGNOdent pen, CarieScan } \\
\text { PRO (CP),VistaCam, DMF } \\
\text { (Decayed-Missing-Filled - } \\
\text { DMF) e outros. }\end{array}$ & $\begin{array}{l}\text { A pesquisa apontou que } \\
\text { existem variações entre os } \\
\text { estudos e a utilização do } \\
\text { sistema ICDAS para cárie }\end{array}$ & $\begin{array}{c}\text { O sistema ideal de } \\
\text { detecção de cárie deve } \\
\text { permitir o diagnóstico } \\
\text { precoce da cárie em } \\
\text { estágio inicial com um } \\
\text { plano de tratamento } \\
\text { adequado. }\end{array}$ \\
\hline $\begin{array}{l}\text { CRUZ, A.I. et } \\
\text { al. (2020) } \\
\text { Revisão } \\
\text { integrativa }\end{array}$ & $\begin{array}{l}\text { Mostrar as novas técnicas } \\
\text { para ajudar na detecção de } \\
\text { lesões incipientes. }\end{array}$ & $\begin{array}{l}\text { Métodos de diagnóstico para } \\
\text { detecção da cárie dental } \\
\text { realizados entre os meses de } \\
\text { maio a junho de } 2020 \text { a partir de } \\
\text { aquisição de dados científicos } \\
\text { das plataformas SciELO, } \\
\text { LILACS e Pubmed. }\end{array}$ & $\begin{array}{l}\text { Após a realização de uma } \\
\text { busca na literatura foram } \\
\text { encontrados dez artigos } \\
\text { relacionados com novos } \\
\text { métodos de diagnóstico para } \\
\text { detecção da cárie dental. }\end{array}$ & $\begin{array}{l}\text { Apesar do surgimento } \\
\text { de novos métodos } \\
\text { promissores, a inspeção } \\
\text { visual permanece como } \\
\text { método de primeira } \\
\text { escolha. métodos devem } \\
\text { ser utilizados como } \\
\text { ferramentas auxiliares. }\end{array}$ \\
\hline $\begin{array}{l}\text { DÜNDAR, A. } \\
\text { et al. (2020) } \\
\text { In vivo }\end{array}$ & $\begin{array}{c}\text { To confirm the detection of } \\
\text { proximal caries using near- } \\
\text { infrared light } \\
\text { transillumination (NILTI) } \\
\text { (DIAGNOcam) device, and } \\
\text { to compare the diagnostic } \\
\text { performance of the device } \\
\text { with other caries detection } \\
\text { methods }\end{array}$ & $\begin{array}{l}\text { A total of } 974 \text { proximal surface } \\
\text { of permanent posterior teeth } \\
\text { from } 34 \text { patients were } \\
\text { evaluated. After clinical } \\
\text { examination of each proximal } \\
\text { surface by an experienced } \\
\text { examiner, they were coded } \\
\text { according to the ICDAS criteria } \\
\text { and subsequently with BW, the } \\
\text { NILTI, LED, and LFpen. }\end{array}$ & $\begin{array}{l}\text { The highest sensitivity } \\
\text { values were recorded from } \\
\text { NILTI readings }(99.1 \%) \text {, } \\
\text { followed by BW scores } \\
(86.8 \%) \text {. The highest } \\
\text { specificity values were } \\
\text { recorded from ICDAS } \\
(100 \%) \text {. The best AUC } \\
\text { values were found from the } \\
\text { NILTI readings }(0.97) \text {. }\end{array}$ & $\begin{array}{l}\text { The NILTI device } \\
\text { exhibited the best } \\
\text { performance in terms of } \\
\text { detecting proximal } \\
\text { dentin caries. }\end{array}$ \\
\hline $\begin{array}{l}\text { HUNG, M. et } \\
\text { al. (2020) } \\
\text { In vitro }\end{array}$ & $\begin{array}{l}\text { In this study, we seek to } \\
\text { investigate the ability with } \\
\text { which } 24 \text { oral and } \\
\text { maxillofacial (OMF) } \\
\text { surgeons assess the } \\
\text { presence of periapical } \\
\text { lucencies on panoramic } \\
\text { radiographs. }\end{array}$ & $\begin{array}{l}\text { The annotation of all panoramic } \\
\text { radiographs took place in } \\
\text { standardized radiology reading } \\
\text { rooms All participating OMF } \\
\text { surgeons annotated the images } \\
\text { on a web-based application, } \\
\text { which was developed for this } \\
\text { study. }\end{array}$ & $\begin{array}{l}\text { Based on the performance } \\
\text { distribution, the probability } \\
\text { that the OMF surgeons had } \\
\text { a PPV greater than } 0.5 \text { was } \\
96( \pm 4) \% \text { whereas the } \\
\text { probability that they had a } \\
\text { TPR greater than } 0.5 \text { was } \\
\text { only } 50( \pm 10) \% \text {. }\end{array}$ & $\begin{array}{l}\text { A deep learning model } \\
\text { trained on a curated data } \\
\text { set of } 2902 \text { de-identified } \\
\text { radiographic images, } \\
\text { can match the mean } \\
\text { diagnostic performance } \\
\text { of } 24 \text { OMF surgeons in } \\
\text { the task of detecting } \\
\text { periodical radiolucent } \\
\text { alterations. }\end{array}$ \\
\hline $\begin{array}{l}\text { SHAN, T et al. } \\
\qquad(2020) \\
\text { Critical review }\end{array}$ & $\begin{array}{l}\text { Outlines the progress and } \\
\text { potential dental applications } \\
\text { of AI in medical-aided } \\
\text { diagnosis, treatment, and } \\
\text { disease prediction and } \\
\text { discusses their data } \\
\text { limitations, interpretability, } \\
\text { computing power, and } \\
\text { ethical considerations, as } \\
\text { well as their impact on } \\
\text { dentists. } \\
\end{array}$ & $\begin{array}{l}\text { Create a backdrop for future } \\
\text { research in this rapidly } \\
\text { expanding arena. }\end{array}$ & $\begin{array}{l}\text { It is necessary to maintain a } \\
\text { proactive attitude toward AI } \\
\text { to ensure its affirmative } \\
\text { development and promote } \\
\text { human-technology rapport } \\
\text { to revolutionize dental } \\
\text { practice. }\end{array}$ & $\begin{array}{l}\text { AI is progressing } \\
\text { rapidly, with potential } \\
\text { applications in } \\
\text { diagnosis, treatment, } \\
\text { and prognosis } \\
\text { predictions. }\end{array}$ \\
\hline
\end{tabular}


Fonte: Autores (2020).

Foram selecionados, dentre os critérios, 12 artigos científicos. Destes, 4 estudos in vitro, 3 estudos in vivo, 1 revisão narrativa, 1 revisão sistemática, 1 revisão de escopo e 1 revisão crítica. Os resultados mostraram o avanço da tecnologia na descoberta e aperfeiçoamento de técnicas para melhorar e aprimorar o diagnóstico da cárie. Dentre essas técnicas, abordamos o infravermelho próximo e as redes neurais convulsionais, embora requeiram um maior número de pesquisas clínicas, em especial randomizadas, com um maior número de pacientes e aplicações para se obter conclusões mais significativa

\section{Discussão}

De acordo com Angelino et al. (2017), Schwendicke et al. (2019), a eficácia da utilização do infravermelho juntamente com a análise de imagens através de redes neurais (CNNS) vem desempenhando um papel importante para o diagnóstico da cárie, uma das doenças crônicas mais prevalentes em todo o mundo e que é capaz de se desenvolver em qualquer fase da vida. O sistema NIR pode ser usado facilmente na prática odontológica diária garantindo inúmeros benefícios, por ser capaz de exibir cáries subterrâneas, incluindo aquelas em torno de obturações, desmineralização, fraturas e rachaduras, tudo sem o uso de radiação ionizante, excelente detalhe das imagens, principalmente em comparação com as radiografias convencionais. Potencialmente concede ao clínico maior poder de um diagnóstico precoce no estágio inicial da doença, além disso, constata-se o baixo custo dos componentes necessários para produzir essas imagens de alta qualidade, que serão avaliadas pelo novo método de algoritmo. Essas ramificações da IA mostram o quanto a tecnologia está progredindo, com aplicações potenciais em previsões de diagnóstico, tratamento e prognóstico, sua relevância e impacto para a sociedade estão relacionados à melhoria na saúde pública, e a garantia de que o cirurgião dentista tenha diversos meios para realizar um método de tratamento adequado e melhorando, também, a tomada de decisão (Araújo et al. 2020; Dündar et al 2020; Shan et al. 2020).

Com relação ao novo âmbito da inteligência artificial, que ao ser estudado por Lee et al. (2018), Endres et al. (2020) demonstrou grande eficiência e maior facilidade, quando utilizada juntamente com o NIR. É chamada de Redes Neurais Convolucionais (CNNS), capaz de analisar as imagens obtidas através do infravermelho e gerar algoritmos de computador que conseguem considerar combinações de variáveis para classificar a presença e ausência de cárie radicular de forma precisa e direta. Ademais, os algoritmos também podem categorizar pacientes que tenham maior risco de desenvolver a cárie radicular e fornecer referências desses pacientes aos profissionais de saúde bucal para avaliação adicional, intervenção precoce e prevenção. Sua relevância e impacto estão associados com um ganho de performance das organizações, o que acarreta em maior produtividade, redução de custos e um desempenho menos suscetível a erros.

Porém, Endres et al. (2020) e Cruz et al. (2020) ressaltam que uma tecnologia relativamente nova no mercado, pode apresentar limitações como a necessidade de um estudo longitudinal no futuro, para estabelecer e confirmar a capacidade preditiva do modelo, necessitando de mais pesquisas e investimento na área para que outros testes sejam realizados a fim de garantir sua eficiência. Mesmo já tendo sido testada e avaliada por diversos profissionais, ainda requer um devido aperfeiçoamento. Apesar disso, é um projeto de extrema qualidade e que tem tudo para se destacar e ser muito utilizado. (Man et al. 2019).

Este estudo se trata de uma revisão integrativa, nele foram analisados estudos relevantes sobre a aplicação das redes neurais na análise de imagens obtidas através do infravermelho. Foi realizada uma síntese dos conhecimentos relacionados a esse assunto, indicando as principais vantagens, desvantagens, dificuldades e necessidades de estudo com relação ao assunto referido, sendo, assim, um suporte em potencial para profissionais de saúde. Este estudo se torna relevante, pois encontramos em sites de grande relevância e confiança, 2 artigo sobre a utilização do Infravermelho (NIR), 3 artigos que se refere a Rede Neural Convolutional e outros 7 artigos que tratam de uma visão menos especifica dessa utilização de inteligência artificial na 
odontologia. $\mathrm{O}$ trabalho foi feito por meio de uma metodologia do tipo qualitativa, utilizando coleta por amostragens, conforme Pereira et al. (2018).

Importante revelar que, ao colocarmos os descritores "Dental Cavity", "Artificial Intelligence", e "Dentistry", encontramos trabalhos que não eram compatíveis com nosso objetivo, o que evidencia a importância da realização de pesquisas dessa natureza, para fortalecer as técnicas da utilização do sistema NIR, juntamente com o algoritmo CNNS, na odontologia. Por sua vez, ao usarmos os bancos de dados, nos deparamos com a dificuldade de encontrar estudos relativos a esse tema, que foi solucionada quando realizamos pesquisas e um refinamento manual, até chegarmos aos artigos que nos eram interessantes. Observamos que foi fundamental o uso de "AND" E "MORE" para afunilar e obtermos tais resultados. Incluímos apenas os artigos completos, pois aqueles que disponibilizam apenas os resumos foram excluídos por não conter dados inteiros das pesquisas. As plataformas usadas foram: Pubmed, Cochrane, Web of Science e Scopus, que são referências mundiais. Além dessas plataformas, também houve uma busca manual, que foi feita através de uma investigação nas referências dos artigos que foram obtidos nas plataformas oficiais citadas acima. Assim, nossos resultados colaboram com a comunidade acadêmica e de clínicos quando apresenta resultados de pesquisa com fontes seguras e uso de descritores cadastrados em banco de dados e plataformas seguras e com credibilidade acadêmica.

É necessário ressaltar também nossa dificuldade para encontrarmos publicações direcionadas ao conteúdo em questão, que por se tratar de um assunto novo ainda enfrenta uma escassez e restrição na quantidade de trabalhos publicados. Porém seu sucesso e reconhecimento promete chegar em um futuro próximo trazendo também mais discussões ao seu respeito nas plataformas renomadas referentes a submissão de artigos. Diante disso, enfrentamos desafios na construção desse projeto, mas fomos motivados pelo desejo de contribuir cada vez mais com a comunidade acadêmica.

Os métodos avançados de detecção da cárie dentária vêm sendo desenvolvidos e complementando-se aos convencionais, o que permite um avanço na detecção de cárie em seu estágio mais precoce (Leão Filho, J. C. B. \& De Souza, T. R. 2017). Em síntese, é possível concluir que tais técnicas (NIR e CNNs) funcionando em harmonia prometem grande êxito e sucesso no seu desempenho, assegurando conforto e tranquilidade para os profissionais, assim como para os pacientes que, por consequência, alcançarão melhor qualidade de vida. (Lalitha. 2019; Man et al. 2019).

\section{Considerações Finais}

Podemos afirmar que a literatura converge quanto à eficácia, praticidade, segurança e aplicabilidade clínica da utilização do Infravermelho, juntamente com as Redes Neurais Convulsionais. Geram benefícios, já que detectam a presença e ausência de cárie radicular de forma precisa e direta.

Quanto a técnica de uso do sistema infravermelho próximo (Near - Infrared, NIR), esta garante imagens de alta qualidade, juntamente com o programa algoritmo de Redes Neurais Convulsionais (CNNS), que oferece um diagnóstico preciso ao analisar tais imagens, e é considerada segura, eficaz, prática e vem demonstrando um grande potencial para seu sucesso no mercado. Além disso, também podemos abordar que o bom desempenho dessa técnica não depende apenas do esforço e da experiência do profissional, mas sim de máquinas treinadas e, por isso, resultam na maior capacidade de entregar o resultado do diagnóstico com precisão, eficiência e agilidade.

Assim, o diagnóstico precoce nas diversas áreas da saúde se torna, a cada dia, uma atitude preventiva de alto impacto para sociedade e, por isso, acreditamos que os investimentos em pesquisa nessa área se tornam relevantes.

Deixamos como sugestão para trabalhos futuros pesquisas associando a ciência de dados, inteligência artificial, uso se sensores, IOT e todos outros mecanismos que possam acelerar entrega de dados precisos aos cuidadores da saúde. Dentre os 
Research, Society and Development, v. 10, n. 4, e43210413083, 2021

(CC BY 4.0) | ISSN 2525-3409 | DOI: http://dx.doi.org/10.33448/rsd-v10i4.13083

estudos na área da saúde bucal que precisam ser aprimorados, vimos o diagnóstico da cárie, das doenças periodontais e do câncer bucal, que poderão garantir eficácia em tratamentos com melhor qualidade e diagnósticos mais precisos.

\section{Referências}

Angelino, K., Edlund, D.A., Shah, P., et al. (2017). Near-Infrared Imaging for Detecting Caries and Structural Deformities in Teeth. (5):2300107. doi: 10.1109 / JTEHM.2017.2695194.

Araújo, A. A., et al. (2020). Métodos de detecção e diagnóstico de cárie: uma revisão narrativa. Research, Society and Development. 9(10), e36291110019. doi: $10.33448 /$ rsd-v9i10.10019.

Cruz, A. I., Gomes, Neto, M. M., Lima, W. T. S., Silva, W. A., Hora, S. L. (2020). Novos métodos de diagnóstico para detecção da cárie dental - Revisão integrativa. Research, Society and Development. 9(10, e7209109160. doi: 10.33448/rsd-v9i10.9160

Dündar, A., Çiftçi, M. E., İşman, Ö., Aktan, A. M. (2020). In vivo performance of nearinfrared light transillumination for dentine proximal caries detection in permanent teeth. The Saudi dental journal, 32(4), 187-193. https://doi: 10.1016/j.sdentj.2019.08.007

Endres, M. G., Hillen, F., Salloumis, M., Sedaghat, A. R., Niehues, S. M., Quatela, O., et al. (2020). Development of a Deep Learning Algorithm for Periapical Disease Detection in Dental Radiographs. Diagnostics (Basel). 10(6): 430. https://doi: 10.3390/diagnostics10060430.

Hung, M., Voss, M. W., Rosales, M. N., Li, W., Su, W., Xu, J., et al. (2019). Application of machine learning for diagnostic prediction of root caries. Web of Science, 36: 395-404. https://doi: 10.1111/ger.12432

Leão, Filho. J. C. B., de Souza, T. R. (2017). Métodos de detecção de cárie: do tradicional às novas tecnologias de emprego clínico. Revista de Odontologia da Universidade Cidade de São Paulo, 23(3), 253-265. Recuperado de http://publicacoes.unicid.edu.br/index.php/revistadaodontologia/article/view/385

Lee, J. H., Kim, D. H., Jeong, S. N., Choi, S. H. (2018). Detection and diagnosis of dental caries using a deep learning-based convolutional neural network algorithm. 77: 106-111. https://doi: 10.1016/j.jdent.2018.07.015

Pereira, A. S., et al. (2018). Metodologia da Pesquisa Cientifica. (e-book). Santa Maria. Ed. UAB/NTE/UFSM.

Pingali, L. (2019). Personal oral health consultant using multimodal machine detection and learning with smartphones and cloud. (10). https://doi: 10.1109/ CCEM48484.2019.000-3

Shan, T., Tay, F. R., Gu, L. (2020). Application of Artificial Intelligence in Dentistry. SAGE Journal, 100(3):232-244. https://doi: 10.1177/0022034520969115

Schwendicke, F., Golla, T., Dreher, M., Krois, J. (2019). Convolutional neural networks for dental image diagnostics: A scoping review. 91: 103226. https://doi: 10.1016/j.jdent.2019.103226 